Sarmiento, J., Garcés. J. (2016). Criptodivisas en el entorno global y su incidencia en Colombia. Revista Lebret, 8. Bucaramanga, Colombia: Universidad Santo Tomás, pp. 151 - 171. ISSN: 2145-5996

\title{
Criptodivisas en el entorno global y su incidencia en Colombia
}

\section{Criptocurrency in the global environment and its impact on Colombia}

Jaime Enrique Sarmiento Suárez ${ }^{l}$

José Luis Garcés Bautista

\begin{abstract}
Resumen
Desde el 2009 cuando surgió la primera criptodivisa no se pensó en el creciente uso de este tipo de "monedas virtuales" como mecanismos financieros alternativos al sistema monetario actual. En la actualidad existen aproximadamente unas 680 criptodivisas, de las cuales el Bitcoin ocupa el primer lugar entre todo este tipo de monedas. En el mundo existe una diversidad de opiniones acerca de las criptodivisas, y varios países ya se han pronunciado al respecto, debido al nivel de apropiación y uso de este tipo de monedas en sus economías. Este artículo surge de la necesidad de conocer la incidencia y el grado de incorporación de las criptodivisas en Colombia, dado que no se tiene ningún tipo de estudio que permita reflejar la realidad colombiana sobre este aspecto, por tal razón, es importante revisar el papel que están jugando las criptodivisas en el entorno global y particularmente en Colombia. Para ello se realizó una encuesta a nueve empresas activas y que actualmente contemplan el uso de criptodivisas como medio de intercambio de bienes o servicios.
\end{abstract}

\section{Palabras clave}

Bitcoin, criptodivisas, monedas virtuales, dinero virtual.

\section{Códigos de clasificación JEL: D12, D84, E41, E51}

\begin{abstract}
Since 2009 when the first cryptocurrency came to the financial system, the increased use of this type of "virtual currency" wasn't given much thought as an alternative of financial mechanisms to the current monetary system. At the moment, there are approximately 680 cryptocurrencies, in which the "Bitcoin" ranks first among all of this type of currencies. In the world there is a diverse amount of opinions on cryptocurrencies and several countries have already spoken about the assumption of using this coin in their economies. This article was born from the need of knowing the influence and degree of incorporation of cryptocurrencies in Colombia, subsequently it doesn't have any study that reflects the Colombian reality on this aspect.
\end{abstract}

\section{Keywords}

Bitcoin, cryptocurrencies, virtual coin, virtual money

* Artículo derivado del proyecto de Investigación: "Criptodivisas en el entorno global y su incidencia en Colombia" Grupo GRICANI Facultad de Administración de Negocios Internacionales, Universidad Pontificia Bolivariana Seccional Bucaramanga.

1 Magíster en Administración. Docente Escuela de Ciencias Estratégicas Universidad Pontifica Bolivariana Seccional Bucaramanga. Correo electrónico: jaime.sarmientos@upb.edu.co.

2 Magíster en Gerencia de Negocios Internacionales, docente Escuela de Ciencias Estratégicas Universidad Pontifica Bolivariana Seccional Bucaramanga. Correo electrónico: jose.garces@upb.edu.co. 


\section{Introducción}

A través del tiempo, en el mundo se ha venido hablado de crear una sola moneda mundial con el ánimo de facilitar las operaciones financieras, eliminar coberturas de divisas y desaparecer el riesgo de guerras monetarias (procesos de devaluación o de depreciación de la moneda con el fin de ser más competitivos). A la fecha esto todavía suena bastante utópico, porque habría que poner en sintonía todos los países en sus aspectos económicos, cosa que no es tarea fácil, y que es bien sabido con la llegada e implementación del euro en Europa.

Las criptodivisas surgen como una alternativa al sistema monetario actual, y tienen como "ventajas" que no se encuentran reguladas por ningún Banco Central, y de cierta manera evita la presencia de intermediarios en el proceso de creación del dinero y las transacciones que con él se realicen, sin el uso de servidores, mediante un esquema $\mathrm{P} 2 \mathrm{P}^{3}$.

Este tipo de monedas tiene sus orígenes a principios del 2009 cuando un enigmático personaje sin identificar, y que firmó con el nombre de Satoshi Nakamoto, lanzó la idea financiera más osada en los últimos tiempos (Nakamoto, 2008), y dio inicio a lo que podría convertirse en la moneda del futuro, el bitcoin, el cual podría convertirse en la moneda única mundial del futuro.

Según la página web (Coinmarketcap, 2016), el número de criptodivisas que existen a la fecha es de 677 , y las principales criptodivisas de acuerdo con su capitalización está dado de la siguiente manera:

Tabla 1. Criptodivisas según su capitalización

\begin{tabular}{rlrrrc}
\hline$\#$ & Name & Symbol & Market Cap (USD) & \% Participation & $\begin{array}{c}\text { \% Cumulative } \\
\text { Participation }\end{array}$ \\
\hline 1 & Bitcoin & BTC & $\$ 6.423 .189 .226,00$ & $85,73 \%$ & $85,73 \%$ \\
\hline 2 & Ethereum & ETH & $\$ 353.167 .709,00$ & $4,71 \%$ & $90,44 \%$ \\
\hline 3 & Ripple & XRP & $\$ 278.641 .833,00$ & $3,72 \%$ & $94,16 \%$ \\
\hline 4 & Litecoin & LTC & $\$ 144.861 .820,00$ & $1,93 \%$ & $96,09 \%$ \\
\hline 5 & Dogecoin & DOGE & $\$ 29.170 .952,00$ & $0,39 \%$ & $96,48 \%$ \\
\hline & Otras & & $\$ 263.414 .823,00$ & $3,52 \%$ & $100,00 \%$ \\
\cline { 2 - 5 } & Total Market Cap & $\$ 7.492 .446 .363,00$ & & \\
\cline { 2 - 3 } & &
\end{tabular}

Fuente: elaboración propia con base en Coinmarketcap (2016).

Se puede observar la predominancia que tiene por el momento el bitcoin, ya que a la fecha cuenta con aproximadamente un $86 \%$ de la participación. Hoy las criptodivisas están marcando una nueva tendencia, y podrán marcar un hito en la economía global.

3 Una red P2P (Peer to Peer) es una red descentralizada, donde los nodos que la conforman se comportan como clientes y servidores, sin la existencia de una jerarquía entre ellos, garantizando que todos son iguales entre sí. 
En el presente trabajo se tomaron las empresas registradas en Colombia en el portal Coinmap con fecha de corte agosto del 2015, y se decidió realizar un censo debido al número pequeño del tamaño de la población. Posteriormente, se realizó una encuesta a todas las empresas que pudieron ser contactadas, así como a dos entidades estatales y a la principal asociación bancaria del país, con el fin de conocer su posición al respecto del uso de criptodivisas en el país. Al final, se encontró que el grado de incorporación de criptodivisas es muy incipiente debido al desconocimiento de estas.

\section{Marco teórico}

\section{Las criptodivisas}

Las criptodivisas son un medio de intercambio digital que, por su diseño y funcionamiento cumplen con las funciones del dinero tradicional permitiendo el intercambio de bienes y servicios. Una criptomoneda se almacena en un monedero electrónico, que le permite realizar las operaciones de compra y venta a que haya lugar. La tasa de cambio está dada por la percepción de valor que le dan los usuarios que las utilizan como mecanismo de transacción, ya que no existe un ente que determine su precio. Las criptomonedas tienen dos implicaciones fundamentales (Díaz, 2014), y es que combinan la comodidad de poder hacer pagos por Internet con el anonimato de los pagos en efectivo.

El inicio de las criptodivisas como tal, se le atribuye a la creación y puesta en funcionamiento del bitcoin, pero esta idea de monedas electrónicas data de 1985, cuando fue propuesta por David Chaum en su artículo "Security without identification: transaction systems to make Big Brother obsolete", donde el autor expresa que el sistema automatizado de transacciones a gran escala del futuro próximo podría ser diseñado para proteger la privacidad y mantener la seguridad de las personas y organizaciones (Chaum, 1985).

El Bitcoin nace a principios del 2009, y su creador fue Satoshi Nakamoto, personaje del cual no se sabe nada, surge como una forma de moneda digital, creada y mantenida electrónicamente, y no se encuentra bajo control de ninguna autoridad. Los bitcoins son producidos por muchas personas que manejan computadores de todo el mundo, usando software que resuelve problemas matemáticos, y es considerado el primer ejemplo de criptomoneda (CoinDesk, 2016).

Desde la creación del bitcoin, han surgido múltiples criptodivisas que se conocen como altcoins, término que se usa para acuñar la abreviatura de "Bitcoin alternativas". La mayoría de altcoins esperan sustituir o mejorar de una u otra forma al menos un componente Bitcoin. 
Dado que la mayoría de criptodivisas se basan en el Bitcoin, es importante conocer cómo es el funcionamiento de esta "moneda virtual". En el sistema Bitcoin hay dos tipos de participantes, por un lado se encuentran los "usuarios" quienes compran y venden usando los bitcoins, generando y publicando las transacciones correspondientes. Por otra parte, se encuentran los mineros, quienes reciben transacciones de otros usuarios, las verifican, y trabajan para incluirlas en la cadena de bloques de transacciones aceptadas (Instituto Nacional de Ciberseguridad, 2014).

La manera como se crean o generan bitcoins es a través de la minería, que es un proceso mediante el cual un grupo de personas a través de computadores especializados contribuyen a la creación de bitcoins, resolviendo problemas matemáticos complejos, que son fáciles de reproducir pero imposibles de revertir y difíciles de predecir, y como recompensa se reciben varias unidades de esta moneda (Bitcoin, 2016).

La creación de bitcoins es predecible y decreciente, cada año el número de bitcoins se reduce a la mitad de forma automática a lo largo del tiempo hasta que la emisión de bitcoin se detenga por completo al llegar al límite de los 21 millones. Se espera que llegado este punto, los mineros de bitcoin sean mantenidos exclusivamente por las numerosas y pequeñas tasas de transacciones. Un bitcoin puede ser dividido en 8 decimales, con los cuales las personas pueden hacer transacciones de bitcoins conocidos como satoshis (Bitcoin, 2016).

Para hacer transacciones con bitcoins solo se requiere tener una billetera virtual, la cual se puede descargar en la página oficial https://bitcoin.org/en/choose-yourwallet, donde se podrá elegir la aplicación para dispositivos móviles, computadores o para un sitio web. Una vez se tenga la billetera se podrá generar una dirección única, la cual se debe compartir para realizar las transacciones a que haya lugar.

Adicionalmente a la clave pública, existe una clave privada que sirve como firma digital y que verifican la identidad y evita que se hagan alteraciones de las transacciones. Las transacciones con bitcoins son verificadas usando un registro público compartido, llamado blockchain, que mantine absolutamente todas las transacciones que se hacen, sin excepción. El blockchain se encarga de asegurarse de que un usuario efectivamente tiene la cantidad de bitcoins que pretende gastar (Blockchainusa, 2014).

\section{Marco global de las criptodivisas}

Desde la creación del bitcoin en el 2009, el uso y la masificación de las criptodivisas ha ido en aumento, por tal razón, algunas naciones ya se han pronunciado al respecto con el ánimo de tomar alguna posición legal frente al tema.

En el 2013, Estados Unidos le dio un espaldarazo muy fuerte a la comercialización de bitcoins, la agencia federal encargada de la supervisión de los mercados financieros 
en Estados Unidos - SEC (Securities and Exchange Commission) y el Departamento de Justicia de este mismo país dijeron ante la Comisión del Senado que el bitcoin es un "medio legal de cambio", y además acotaron que las monedas virtuales en sí mismas, no son ilegales (Raskin, 2013).

China prohibió a los bancos transar con bitcoins pero los usuarios pueden comprarlas y venderlas por internet, mientras no se toque la banca. En Singapur, el sistema no se ve como una moneda sino como una mercancía que igual se compra y se vende y por tanto debe pagar impuestos (Gómez, 2014)

En Japón se determinó que no es una moneda, pero se analiza gravarlo como un valor (Gómez, 2014), por el momento no tienen la intención de legislar sobre Bitcoin, permitiendo que nueva tecnología florezca y que esta determine su propio camino hacia la regulación. Con ese fin, el país asiático ha anunciado la creación de la Autoridad de Japón de Activos Digitales, con apoyo explícito del Gobierno, y que reunirá a las empresas Bitcoin del país, con el fin de establecer las normas y códigos de conducta para sus miembros. La nueva entidad propondrá directrices y "suavemente monitoreará" a sus miembros, sin legislación necesaria.

En Argentina, la Unidad de Información Financiera (UIF) publicó el 10 de julio de 2014 una nueva normativa en la que se obliga a las entidades financieras (bancos, agencias de cambios, sociedades de bolsa) que operan en el país a informar mensualmente sobre todas las transacciones realizadas con monedas virtuales. Esta nueva norma, pretende, según la agencia, evitar el lavado de dinero y la financiación de terrorismo (Oro y finanzas, 2014).

En Francia, el ministro de Finanzas anunció que su Gobierno ha decidido limitar el anonimato en la compra bitcoins, al imponer que el dueño de una cuenta sea identificado y que se compruebe su identidad en la retirada y entrega de monedas. También propone una cantidad máxima en el pago con monedas virtuales, "justificado por el carácter anónimo de ese tipo de pago y en coherencia con la reglamentación actual que afecta a los pagos en efectivo". Su anuncio tuvo lugar tras haber recibido un informe del organismo gubernamental de lucha contra el blanqueo, Tracfin, en el que se recalca que aunque el volumen existente de bitcoins no es susceptible de desestabilizar el sistema financiero, el desarrollo de esas monedas no oficiales presenta riesgo de uso ilícito (Oro y finanzas, 2014).

En Ecuador, la Asamblea Nacional prohibió el uso de bitcoin y de otras monedas digitales descentralizadas, y estableció al mismo tiempo la creación de una nueva moneda electrónica, la estatal (Higgins, 2014).

En Bolivia, el 06 de mayo de 2014 el Banco Central de dicho país emitió la Resolución de Directorio 044/2014 que prohíbe expresamente el uso de monedas no emitidas o regulados por los Estados, entre las que figuran el bitcoin y una larga lista de criptodivisas. El artículo 1 de esta Resolución resuelve que: 
A partir de la fecha queda prohibido el uso de monedas no emitidas o reguladas por estados, países o zonas económicas, y de órdenes de pago electrónicas en monedas y denominaciones monetarias no autorizadas por el Banco Central de Bolivia en el ámbito del sistema de pagos nacional (Banco Central de Bolivia, 2014).

En Brasil, se promulgó la Ley 12.865 del 09 de octubre de 2013, que creó la posibilidad de la normalización de los sistemas de pago móvil y la creación de monedas electrónicas (Normas Brasil, 2013). De acuerdo con la Receita Federal do Brasil, los bitcoins equivalen a activos financieros para efectos tributarios y, por eso, deben ser declarados como "otros bienes" por quien posea el equivalente a R\$ $1.000,00$ o más en 2013. También es necesario pagar Impuesto de Renta de un 15\% sobre la ganancia de capital en transacciones superiores a R \$ 35 mil (Patricia Peck Pinheiro Abogados, 2014).

Con relación a la Unión Europea, ellos consideran las critodivisas como legales, y adicionalmente el Tribunal de Justicia de la Unión Europea el 22 de octubre de 2015 dictó una sentencia donde dejó claro que el bitcoin y otras monedas virtuales están exentas de IVA (InfoCuria, 2015). Dentro de la sentencia se hace referencia a que el Banco Central Europeo en el 2012 indica que una divisa virtual se define como un tipo de moneda digital no regulada, emitida y verificada por sus creadores y aceptada por los miembros de una comunidad virtual concreta, y que particularmente el bitcoin es una divisa virtual denominada de "flujo bidireccional" que los usuarios pueden comprar y vender con arreglo al tipo de cambio. Por lo que respecta a su uso en el mundo real, estas divisas virtuales son análogas a las demás divisas intercambiables, y permiten adquirir bienes y servicios tanto reales como virtuales.

Por su parte, en Colombia la Superintendencia Financiera emitió la Carta circular 29 de marzo 26 de 2014, con el fin de que el público en general conozca y entienda los riesgos a los que se exponen cuando adquieren y transan con monedas virtuales, las cuales no están reguladas, ni respaldadas por ninguna autoridad monetaria o activos físicos, y cuya aceptación es muy limitada, pero en ningún aparte dice que son ilegales (Superintendencia Financiera de Colombia, 2014)

\section{Principales hitos en el precio del bitcoin}

Existen diferentes momentos en el tiempo que han marcado hitos importantes en la evolución del bitcoin, los cuales han servido de marco de referencia para la creación de otras criptodivisas (History of Bitcoin, 2012). En agosto de 2008 se registra el dominio bitcoin.org y en octubre del mismo año se publica el primer documento que explica el diseño del bitcoin, llamado "Bitcoin: A Peer-to-Peer Electronic Cash System". En noviembre el proyecto Bitcoin es registrado en SourceForge.net. En enero del 2009 nace la red Bitcoin con el lanzamiento del primer código abierto de un cliente bitcoin, y posteriormente su creador Satoshi 
Nakamoto mina el primer bloque de bitcoins, por el cual recibe 50 bitcoins, conocido como bloque génesis, a partir de ese momento han presentado diferentes hitos que han ido marcando la evolución del precio de los bitcoins.

La primera transacción de bitcoin se realiza en enero de 2009 entre Satoshi y un desarrollador llamado Hal Finney. En octubre del mismo año, la organización New Liberty Standard publica la tasa de cambio del bitcoin, la cual la establece en US\$1 = 1.309.03 BTC, y la determina usando una ecuación que incluye el costo de la electricidad que utiliza un computador para generar bitcoins (Bitcoinwiki, 2015).

En mayo de 2010 se hace la primera transacción real, cuando un programador de Jacksonville, Florida, llamado Laslo Hanyecz, ofrece pagar 10.000 bitcoins por una pizza en el Foro de Bitcoin. Él envió las bitcoin a un voluntario en Inglaterra, quien realizó la orden a través de su tarjeta de crédito. En ese momento, el tipo de cambio puso el precio de compra de la pizza en torno a US\$ 25 (Bitcoinx, 2014).

Durante el mes de julio de 2010, el valor de cambio del bitcoin incrementa diez veces, pasando de US\$0.008/BTC a US\$0,08/BTC. En el mismo mes se crea el sitio de intercambio de bitcoins llamado Mt.Gox. Este sitio llegó a manejar aproximadamente el $90 \%$ de las transacciones de bitcoin.

En agosto de 2010 una vulnerabilidad del sistema Bitcoin es descubierta y se emiten 184 mil millones de bitcoins en una sola transacción. En cuestión de horas la transacción es detectada, borrada y la vulnerabilidad arreglada. A la fecha es la única vulnerabilidad detectada en el protocolo Bitcoin. En septiembre de 2010 se utiliza un método en el cual varios usuarios trabajaban colectivamente para minar Bitcoin y compartían los beneficios, este método se llamó "Bitcoin Pooled Mining" (History of Bitcoin, 2012).

En octubre de 2010, el Grupo de Acción Financiera (Financial Action Task Force), un grupo intergubernamental, creado en 1989 y conformado por 35 países miembros, y que tienen como función desarrollar y promover políticas para evitar el lavado de dinero y la financiación de los terroristas, publica sobre el blanqueo de dinero a través de nuevos métodos de pago, para advertir sobre el uso de las monedas digitales para financiar grupos terroristas.

En noviembre de 2010, el valor de los Bitcoin emitidos supera el millón de dólares. Este cálculo se basó en la multiplicación del número de bitcoins en circulación por el valor de la última operación en Mt.Gox. Para finales del 2010, el valor del bitcoin cierra a USD $\$ 0.30$.

En febrero del 2011, el mercado bitcoin llamado la Ruta de la Seda (Silk Road) abre sus puertas; funcionaba como una plataforma de mercado ilícito para la comercialización de droga. Era llamado el eBay de las drogas. En el mismo mes, el bitcoin alcanza la paridad con el dólar, y adicionalmente un miembro australiano del Foro Bitcoin intenta vender su vehículo Celica Supra 1984 por 3.000 BTC, y se 
convierte en la primera persona en ofrecer un vehículo a cambio de bitcoins (History of Bitcoin, 2012).

En marzo de 2011, es vendido Mt.Gox a la empresa Tibanne Company de Japón. En el mismo mes Bitcoin GB se abre y se convierte en el primer mercado para el intercambio de bitcoins por libras esterlinas, así como en Brasil aparece Bitcoin Brasil y se convierte en el primer mercado de intercambio de bitcoins por reales brasileros.

En abril de 2011, Bitmarket.eu abre y se convierte en el primer mercado de intercambio de bitcoins por euros y otras monedas de Europa. En este mes, se realiza la primera publicación en los medios sobre bitcoin, la revista TIME publica un artículo sobre esta criptodivisa llamado "Online cash Bitcoin could Challenge Governments, Banks". Adicionalmente, la tasa de cambio del bitcoin alcanza y supera la paridad con el euro y la libra esterlina. El valor de los bitcoin emitidos supera los diez millones de dólares (Tecnología Bitcoin, 2014).

En junio de 2011, la tasa de cambio alcanza el valor de US\$10/BTC y sube hasta US\$31,91/BTC. Cuatro días después se desplomó el precio a cerca de US\$10/BTC, este incidente se conoció como la Gran Burbuja del 2011. También en este mes un miembro del Foro Bitcoin, reportó que 25.000 BTC fueron robados de su cartera electrónica. Por otra parte, WikiLeaks comienza a aceptar donaciones anónimas de Bitcoins (Europapress, 2011).

Otro hecho importante que también se dio en junio de 2011, es que Mt.Gox, el broker que en aquel momento realiza el 90\% del volumen de transacciones de compra venta de bitcoins sufre su primera gran vulnerabilidad, 600 de sus clientes ven cómo sus cuentas son robadas y una gran cantidad de órdenes de venta hace que el precio del bitcoin se desplome de USD\$17,1 a USD\$0,01. Toda operación de compra-venta en Mt.Gox queda paralizada durante 7 días. En este mismo mes, la Fundación Fronteras Electrónicas (Electronic Frontier Foundation - EFF) cesa la aceptación de donaciones en bitcoins por razones de inseguridad jurídica. De otra parte, la empresa BitPay lanza su primer monedero electrónico (e-wallet) de bitcoins para teléfonos inteligentes. En diciembre del 2011 el bitcoin cierra a un valor de USD $\$ 4,72$ (Criptoeconomia, 2015).

En febrero del 2012 aparece la primera revista cuya temática es el bitcoin (Bitcoin Magazine). En marzo de 2012, debido a un fallo de seguridad en Linode, una empresa de alojamiento de sitios web, se produce el mayor robo de bitcoins registrado a la fecha, más de 46.000 BTC son robados, y estaban valorados en más de US\$228.000. En abril de 2012 se filtró un informe del FBI, donde manifestaban la preocupación de que los métodos de pago como el bitcoin podrían facilitar las transacciones ilegales de armas y narcóticos. En septiembre de 2012, Bitfloor, la cuarta mayor casa de cambio de bitcoin fue hackeada y perdió 24.000 BTC, valorados en aproximadamente US\$250.000 (Siles, 2014). 
En noviembre de 2012, el sitio web WordPress anuncia que acepta bitcoins como forma de pago para los usuarios que compren mejoras (upgrades). En diciembre de 2012 la primera casa de intercambio en ser licenciada como un banco europeo es Bitcoin Central, operando dentro del marco regulador europeo. Este mes el Bitcoin cierra a USD \$13,51 (History of Bitcoin, 2012).

En febrero de 2013, Mateo Birkenshaw y Riley Alexander, fundadores de GiftsForCoins.com, comienzan una nueva puesta en marcha, PizzaForCoins.com, un servicio que actúa como un intermediario para permitir a los usuarios ordenar pizza con bitcoins a cambio de una pequeña cuota BTC. También es en este mes Kim Dotcom anuncia que su servicio de almacenamiento en la nube, Mega, comenzará a aceptar pagos a través de Bitcoin bitvoucher.co, su nuevo distribuidor. El valor comercial de un bitcoin alcanza los US\$ 30 por primera vez desde el 2011 (Tecnología Bitcoin, 2014).

En marzo de 2013, la Red de Control de Crímenes Financieros (Financial Crimes Enforcement Network - FinCEN) del Departamento del Tesoro de los Estados Unidos publica su Reglamento de Aplicación de la FinCEN para las personas que administren, intercambien, o utilicen divisas virtuales, definiendo su posición sobre las monedas virtuales. El bitcoin alcanza un valor de US\$ 74.90 (Bitcoinx, 2014).

En abril de 2013, el valor del bitcoin supera los US\$100 y alcanza un máximo histórico de US\$266, en comparación con su valor de US\$13 el año anterior. En mayo de 2013 se estrenó, en San Diego, California, el primer cajero automático de bitcoin en el mundo. En este mes PrimeDice.com lanza una plataforma de casino en línea que acepta apuestas bitcoin (Tecnología Bitcoin, 2014).

En junio de 2013, BitcoinWebHosting.net lanza para ofrecer soluciones de alojamiento web para el desempeño de la comunidad bitcoin, que ofrece múltiples planes de hosting, registro de dominios y protección contra ataques DDoS, mediante el pago de Bitcoin. En agosto de 2013, Bitcoin llega a la terminal de Bloomberg para que los empleados de Bloomberg puedan realizar seguimiento al valor de las BTC bajo el nombre de moneda XBT, que obedece a una norma de la Organización Internacional de Normalización (ISO) que mantiene la lista de las monedas de los países, y que especifica que si una moneda no está asociada a un país comienza con la letra "X" (Euribor, 2014).

En agosto de 2013, el Ministerio Federal de Hacienda Alemán reconoce al bitcoin como una forma de dinero privado, o una «unidad de cuenta», y permite su uso en ventas comerciales y privadas. Bitcoins se convierte en exenta de impuestos cuando se mantiene durante más de un año (Bitcoinx, 2014).

En octubre de 2013, el FBI cierra el famoso mercado de drogas en línea llamado "Ruta de la Seda - Silk Road", se incautaron 3.6 millones de dólares en bitcoins. En este mes, Baidu, el mayor motor de búsqueda de China se convierte en el primer servicio de este tipo en aceptar bitcoin. Por otra parte, BitMarket.eu cierra sus puertas (El Diario, 2013). 
En noviembre de 2013, el precio del bitcoin rompe el record establecido en abril de 2013, llegando a los UD\$269, al alcanzar durante el mismo mes un valor máximo de US\$503.10. Adicionalmente, un restaurante Subway en Allentown, Pennsylvania comienza a aceptar bitcoin, convirtiéndose en el primer Subway en los Estados Unidos en hacerlo (Coindesk, 2013).

En el mismo mes de noviembre de 2013, Estados Unidos le dio un espaldarazo muy fuerte a la comercialización de bitcoins, la Agencia Federal encargada de la supervisión de los mercados financieros en Estados Unidos - SEC (Securities and Exchange Commission) y el Departamento de Justicia de este mismo país dijeron ante la Comisión del Senado que el bitcoin es un "medio legal de cambio", y además acotaron que las monedas virtuales en sí mismas, no son ilegales, esto hace que el precio del bitcoin marque un récord al alcanzar los US\$1.242. El volumen de transacciones de bitcoin supera a Western Union 51 (History of Bitcoin, 2012).

Otros sucesos que se dan en noviembre de 2013, es que la Universidad de Nicosia en Chipre se convierte en la primera universidad que acepta bitcoin para la matrícula, Virgin Galactic empieza a aceptar bitcoin para los viajes espaciales. Shopify integra oficialmente bitcoin como una opción de pago para sus 70.000 comerciantes. Bitcoin Viernes Negro organiza el primer evento anual del Viernes Negro para resaltar las ofertas de los comerciantes y los comercios que aceptan bitcoin (Tecnología Bitcoin, 2014).

En diciembre de 2013, sucede el mayor robo de toda la historia, 96.000 bitcoins son robados del Sheep Marketplace, un sitio en línea de drogas. Además, en este mes el Banco Central de China prohíbe a las instituciones financieras las transacciones con bitcoin, y provoca una caída de los precios del bitcoin, llegando por debajo de los US\$600. Baidu para de aceptar bitcoins después de la prohibición China (Edwards, 2013).

En febrero de 2014, la compañía Mt.Gox suspendió la negociación de bitcoin, cerró su servicio web y se declaró en bancarrota. En abril de 2014 la empresa entró en proceso de liquidación. Se anunció que aproximadamente 850.000 bitcoins que pertenecían a los clientes y a la compañía probablemente habían sido robados, la cantidad estaba valorada en más de US\$450 millones. Debido al robo sufrido por Mt.Gox, el precio de la divisa alcanzó a llegar a los US\$200 (Coindesk, 2013).

Durante el 2014, el bitcoin alcanzó a perder aproximadamente el 60\% del valor con el cual inició ese año, y por tal razón no se consideró como una buena alternativa de inversión durante ese periodo de tiempo. Su valor cerró a diciembre 31 en aproximadamente US\$315 (El Bitcoin, 2015).

En el 2015 presentó una recuperación de su valor, y alcanzó un incremento en su precio en aproximadamente un $36 \%$, cerrando el año en un valor de US\$430. A la fecha, durante el primer trimestre del 2016 no ha presentado un aumento significativo en su valor y su precio se mantiene alrededor de los US\$430. 
El comportamiento del precio de mercado del bitcoin en dólares durante el tiempo de vida de la divisa se puede observar en la siguiente gráfica extraída del sitio web blockchain.info, donde se puede observar el comportamiento volátil de la criptodivisa y cómo algunos aspectos citados anteriormente han influido en la variación del precio durante la existencia de la moneda.

Figura 1. Precio del mercado de bitcoin en dólares

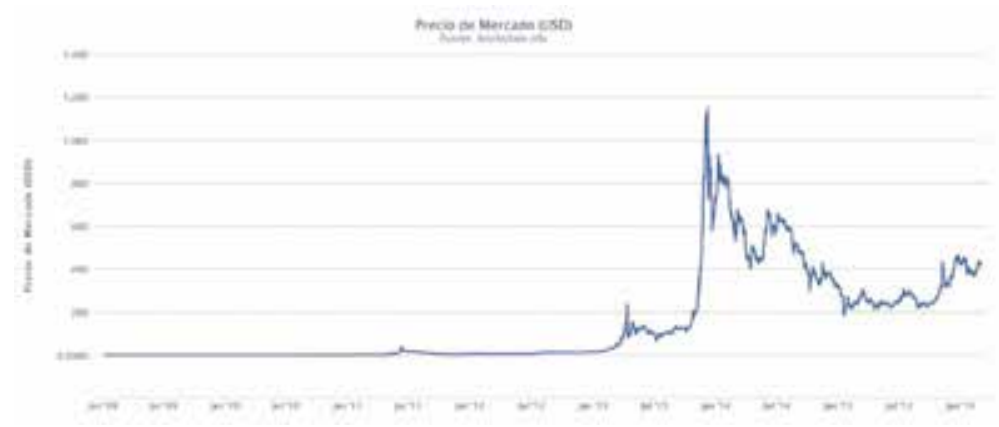

Fuente: (Blockchain, 2016).

\section{Principales criptodivisas}

Según la página web (Coinmarketcap, 2016), el número de criptodivisas que existe a la fecha es de 698, y las principales criptodivisas de acuerdo con su capitalización está dado de la siguiente manera:

Tabla 2. Capitalización del mercado de criptodivisas

\begin{tabular}{llrrrc}
\hline$\#$ & \multicolumn{1}{c}{ Name } & Symbol & Market Cap (USD) & \% Participation & $\begin{array}{c}\text { \% Cumulative } \\
\text { Participation }\end{array}$ \\
\hline 1 & Bitcoin & BTC & $\$ 6.254 .117 .400,00$ & $79,92 \%$ & $79,92 \%$ \\
\hline 2 & Ethereum & ETH & $\$ 803.481 .021,00$ & $10,27 \%$ & $90,18 \%$ \\
\hline 3 & Ripple & XRP & $\$ 278.602 .020,00$ & $3,56 \%$ & $93,74 \%$ \\
\hline 4 & Litecoin & LTC & $\$ 142.059 .769,00$ & $1,82 \%$ & $95,56 \%$ \\
\hline & Otras & & $\$ 347.502 .872,00$ & $4,44 \%$ & $100,00 \%$ \\
\cline { 2 - 3 } & Total Market Cap & $\$ 7.825 .763 .082,00$ & & \\
\cline { 2 - 3 } & & & &
\end{tabular}

Fuente: elaboración propia con base en Coinmarketcap, 2016.

Estas cuatro criptodivisas conforman aproximadamente el $96 \%$ de la capitalización del mercado. A continuación se presentan algunas de las principales características de estas criptodivisas:

- Bitcoin

Tiene un tope de generación de unidades de 21 millones. A la fecha se han creado 15 millones de BTC. El tiempo de espera para confirmar una transacción es de 10 
minutos, si bien es cierto el pago es casi instantáneo, existe un retraso de 10 minutos antes de que la red empiece a confirmar la transacción al incluirla en un bloque y antes de que se puedan gastar los bitcoins recibidos. Una confirmación significa que hay un consenso en la red en que los bitcoins recibidos no han sido enviados a alguien más y son ahora de propiedad de quien los recibió. Una vez que la transacción ha sido incluida en un bloque, esta irá siendo "enterrada" con más confirmaciones por los siguientes bloques que van añadiéndose a la cadena, lo que hará consolidarse este consenso y disminuir el riesgo de revocar la transacción. Cada usuario es libre de determinar en qué punto se puede considerar una transacción como confirmada, pero normalmente 6 confirmaciones es considerado tan seguro como esperar 6 meses tras un pago con tarjeta de crédito (Bitcoin.org, s.f.).

\section{- $\quad$ Ethereum}

Ethereum no surge solo como una criptomoneda, su creador Vitalik Buterin ideó una red de criptomoneda que tiende a ser lo más generalizada posible. Se trata de un sistema operativo que permite a los usuarios crear aplicaciones basadas en monedas virtuales que pueden ir más allá del simple uso financiero. Unas monedas que quedan al gusto y la imaginación de su consumidor. Así, si una persona decide montar una aplicación para hacer transacciones, o lo que es lo mismo, enviar dinero a otras personas, podrá crear una moneda virtual propia a la medida del servicio que pretende ofrecer.

Las monedas inventadas por los usuarios conforman lo que se denomina "monedas de colores". Pero el atractivo de Ethereum no reside en esas monedas, sino en el contrato que crea el sistema una vez que abrimos la nueva aplicación. La web Coin Desk explica que un contrato es un agente autómata que crea el sistema Ethereum para recibir y enviar transacciones, y guardar los balances de esas transacciones.

Con los contratos que crea Ethereum se podrían implementar derivados financieros, ligados a diferentes contratos, para que las transacciones complejas no solo sean posibles, sino también relativamente fáciles. Los portales de juegos online, las predicciones de mercados y la industria aseguradora son algunos de los negocios que podrían beneficiarse del contrato virtual (Chacón, 2014).

- Ripple

Ripple es un sistema de emisión y gestión del crédito basado en una red P2P, donde cada uno de sus integrantes funciona como un banco autónomo con la capacidad de extender y recibir crédito (nominado en diferentes monedas), y de hacerlo circular. Es decir, cada uno de los nodos pertenecientes a la red de Ripple funciona como si fuese una entidad de crédito que presta servicio a otros nodos o usuarios con los que mantiene una relación de confianza, es decir, se realizan transacciones de crédito (intercambio de fondos o préstamos) dentro de un círculo de usuarios con los que se mantiene una relación de confianza (sabemos que podemos "comerciar" con ellos). Al existir esta relación de confianza entre los participantes en la red, la 
suma de todas estas conexiones genera una red de usuarios y "entidades" entre las cuales fluye el crédito y, por tanto, entre las que se pueden realizar transacciones económicas (Velasco, 2013).

JP Koning (El Bitcoin, 2013) acierta al comparar a Ripple con el sistema de letras de cambio que prosperó especialmente en los siglos XVII y XVIII. Estos papeles eran emitidos como un compromiso de pago a cambio de algún producto, y luego eran endosados por una larga cadena de comerciantes antes de alcanzar su madurez. Finalmente, el emisor cumplía con su promesa de pagar en oro el valor nominal de la letra de cambio. Ripple tiene sin duda un gran potencial como instrumento facilitador de los intercambios. Es importante tener en cuenta que bitcoin funciona como una moneda y ripple como un crédito, lo que los hace de cierta manera diferentes, debido a que la primera funciona con base en reglas abstractas y la segunda involucra relaciones y acuerdos personales (El Bitcoin, 2013).

- Litecoin

Basada en el protocolo Bitcoin, pero difiere de este, ya que puede ser extraída con hardware para el consumidor. Brinda confirmaciones de transacciones de manera más rápida, 2.5 minutos en promedio y utiliza un algoritmo de minería orientado a computadores regulares que poseen la mayor parte de las personas. La red Litecoin está programada para producir 84 millones de unidades LTC (Litecoin.org, 2011).

Según la página oficial de Litecoin https://litecoin.org/es/, dice que tiene una ventaja sobre el bitcoin en relación con la cadena de bloques, ya que es capaz de administrar un mayor volumen de transacciones que su homólogo Bitcoin, debido a que la generación de bloques se realiza de manera más frecuente, la red sustenta más transacciones sin la necesidad de modificar el software en el futuro. Como resultado, los comerciantes obtienen confirmaciones de manera más rápida, y al mismo tiempo tienen la posibilidad de esperar más confirmaciones al vender artículos más costosos.

\section{Ventajas y desventajas de las criptodivisas}

Entre las ventajas de las criptodivisas de acuerdo con las características que este tipo de monedas tiene, se pueden citar:

- Reguladas por la oferta y la demanda.

- Se realizan directamente las transacciones entre las partes, no requiere intermediarios.

- No están reguladas por un banco central.

- Los usuarios pueden minar las monedas virtuales.

- Es una nueva alternativa de realizar transacciones financieras.

- No operan como contratos o créditos virtuales, ya que el valor de la criptodivisa 
no depende de la capacidad de un tercero para cumplir con los compromisos que se han contraído.

En relación con las desventajas se tiene:

- Incertidumbre.

- Alta volatilidad.

- Puede prestarse para actividades ilícitas (lavado de dineros, tráfico de drogas, entre otras.)

- En tecnología todo puede suceder.

\section{Metodología}

Este trabajo es un estudio descriptivo y tiene por objetivo presentar la evolución de las criptodivisas en el mundo y su incidencia en Colombia, para ello se recopiló información relacionada con la distribución en peso de las monedas virtuales existentes más representativas y se tomó cuál ha sido la posición de diferentes países con relación al uso de las criptodivisas en sus economías. Para ello fue necesario realizar la recolección de datos mediante el acceso a fuentes primarias y secundarias de información, las cuales incluyeron, entre otros, artículos académicos, consultas a sitios web especializados en información de capitalización de las criptodivisas, sitios web de registro de empresas que aceptan como medio de pago las criptodivisas, blog temáticos.

Particularmente para el caso de Colombia, se realizó la búsqueda de los lugares que se encuentran registrados y que aceptan criptodivisas como medio de pago, se creó una base de datos y se aplicó una encuesta a toda la población objeto de estudio. Adicionalmente, se contactó a entidades gubernamentales y a la principal asociación bancaria, que están relacionadas con el tema, con el fin de conocer su posición al respecto del uso de criptodivisas en el país.

El período de recolección de la información mediante encuestas telefónicas y por correo electrónico a los diferentes actores se realizó durante el segundo semestre del año 2015, tanto a las empresas que manejaban criptodivisas (para ello se realizó una encuesta a nueve empresas activas y que actualmente contemplan el uso de criptodivisas como medio de intercambio de bienes o servicios) así como se consultó a la Superintendencia Financiera, el Banco de la República y Asobancaria con el fin de determinar si su posición frente a las criptodivisas había variado.

\section{Resultados}

No existe una certeza de cuántos lugares están aceptando cualquiera de estas criptodivisas, y dado que el bitcoin se convierte en la criptodivisa más usada a la fecha 
y la que tiene un mayor peso en el mercado, se decidió trabajar con esta criptomoneda y establecer su grado de incidencia en Colombia, para ello tomamos como referencia el sitio web http://coinmap.org/ en el cual se puede visualizar que a marzo 18 de 2016 se encontraban registrados 7640 lugares en el mundo que aceptan transacciones con bitcoin. En Colombia a agosto de 2015 aparecen registrados 25 lugares, los cuales se encuentran distribuidos por ciudad de la siguiente manera (Coinmap, 2016):

Tabla 3. Número de compañias que usan criptodivisas en Colombia según la ciudad

\begin{tabular}{cc}
\hline Ciudad & \# Empresas \\
\hline Bogotá & 12 \\
\hline Medellín & 7 \\
\hline Cartagena & 2 \\
\hline Cali & 2 \\
\hline Cúcuta & 1 \\
\hline Bucaramanga & 1 \\
\hline
\end{tabular}

Fuente: elaboración propia con base en Coinmap, 2016.

Por otra parte, haciendo un chequeo general dentro del mismo sitio web (Coinmap) del tipo de actividad de estos lugares registrados en Colombia, aparecen comercializadoras de bitcoins, hoteles, servicio de café internet, teatro, café-bar, litografía, audiovisuales y producción de video, muffins, entre otras.

Con base en las veinticinco empresas registradas se procedió a realizar una búsqueda y depuración de la información, con el objetivo de determinar a cuáles de ellas se les podría aplicar la encuesta diseñada, de este proceso se obtuvo la siguiente información:

Tabla 4. Clasificación de las empresas según la información obtenida

\begin{tabular}{ll}
\hline \multicolumn{1}{c}{ Descripción } & Cantidad \\
\hline Empresas que se contactaron y respondieron la encuesta & 11 \\
\hline Empresas que no respondieron la encuesta & 2 \\
\hline Empresas con las que no se pudo establecer contacto & 3 \\
\hline Empresas que no existen o no se encuentras activas & 6 \\
\hline Empresas que manifestaron no haber usado criptodivisas & 3 \\
\hline
\end{tabular}

Fuente: elaboración propia.

Después de depurar la información, se encontró que once (11) empresas, las cuales representan el $44 \%$ de las empresas registradas, se encuentran activas y tenían como opción usar criptodivisas en sus transacciones financieras, y que el número de 
ciudades donde se encuentran estas empresas pasan de 6 a 4 (se eliminó Bucaramanga y Cartagena), siendo Bogotá la ciudad que tiene más empresas que ofrecen este tipo de servicio.

Tabla 5. Número de compañias activas que usan criptodivisas en Colombia

\begin{tabular}{cc}
\hline Ciudad & \# Empresas \\
\hline Bogotá & 6 \\
\hline Medellín & 3 \\
\hline Cali & 1 \\
\hline Cúcuta & 1 \\
\hline
\end{tabular}

Fuente: elaboración propia.

Tomando estas once (11) empresas, se les preguntó quienes actualmente tienen las criptodivisas como medio de pago, y dos empresa manifestaron que ya no la consideran como una opción de pago, ya que desde el tiempo en que la implementaron nunca recibieron ninguna transacción de este tipo, y por tal razón, ya no ofrecen este tipo de servicio. De esta manera, solo quedaron realmente nueve (9) empresas activas y que contemplan el uso de criptodivisas.

Se les preguntó qué tipos de criptodivisas reciben y el 100\% respondió que utilizan el bitcoin, una de las nueve empresas adicionalmente también recibe litecoin. También se les preguntó a las empresas desde cuándo reciben o usan el bitcoin y se obtuvo la siguiente información:

Tabla 6. Fecha de implementación de bitcoin por las compañias colombianas

\begin{tabular}{cc}
\hline Año & \# Empresas \\
\hline 2012 & 1 \\
\hline 2013 & 4 \\
\hline 2014 & 2 \\
\hline 2015 & 2 \\
\hline
\end{tabular}

Fuente: elaboración propia.

Esta tabla nos permite evidenciar que es una tendencia joven en el país, ya que las empresas solo llevan 4 años implementando este tipo de prácticas, y que su boom estuvo en el 2013, con aproximadamente un 44\% de las empresas que comenzaron a implementar el bitcoin como una nueva forma de pago. El tamaño de las empresas que predomina son las microempresas con un $91 \%$ y el porcentaje restante corresponde a una pequeña empresa, lo anterior está determinado por el número de empleados. 
Es importante mencionar lo siguiente: las dos empresas de Medellín que tienen el servicio de pago activo de bitcoin, el cual fue implementado por una misma persona que trabaja en ambas organizaciones, y fue quien impulsó este tipo de servicio. Por otra parte, la empresa de Cúcuta y una de Bogotá, están conformadas por dos hermanos, y uno de ellos se encargó de motivar al otro para que implementara este servicio.

Cabe señalar que de las nueve empresas que tienen el servicio de transacción mediante el uso bitcoin, una es una comercializadora de la criptodivisa, es decir, su negocio es la compra y venta de bitcoin, luego su negocio es netamente regido por este tipo de transacciones.

Con el fin de establecer para las ocho empresas restantes la frecuencia de uso de bitcoin, por parte de los clientes y su porcentaje en las ventas mensuales, se obtuvo la siguiente información:

Tabla 7. Frecuencia de uso de "bitcoin"

\begin{tabular}{lcc}
\hline Frecuencia de uso & \# Empresas & \% Ventas mensuales \\
\hline Aún no lo han usado & 3 & $0 \%$ \\
\hline Entre 1 y 5 veces desde la implementación & 3 & $0 \%$ \\
\hline Cada 3 meses & 1 & $1 \%$ \\
\hline Mensualmente & 1 & $4 \%$ \\
\hline
\end{tabular}

Fuente: elaboración propia.

Lo anterior nos permite observar lo incipiente de este tipo de transacciones, ya que un $37,5 \%$ de las empresas aún no han recibido un pago en esta criptodivisa, el otro $37,5 \%$ ha recibido esporádicamente pago con bitcoin, y solo un $25 \%$, recibe de manera "periódica" pago con criptodivisas.

Por último, se les preguntó a las nueve empresas si estaban interesadas en seguir ofreciendo el bitcoin como medio de pago, y el 100\% respondió que sí, y entre las razones que argumentaron fueron:

- Es una tendencia mundial.

- Permite la internacionalización.

- No hay costo de implementación.

- Menor costo en las transacciones.

- Innovador y con mucho potencial.

De otra parte, se buscó la opinión de dos entidades gubernamentales, la Superintendencia Financiera y el Banco de la República, y de un ente privado que 
reúne las entidades bancarias, llamada la Asobancaria ${ }^{4}$, con el fin de conocer si la opinión que tienen acerca de las criptodivisas en Colombia había variado, dado que en otros países del mundo la posición frente a las criptodivisas ha ido evolucionando.

La Superintendencia Financiera, informó que de acuerdo con sus facultades legales, susconsideracionesconrespectoalascriptodivisas, seencuentrancontenidas en la Carta Circular 029 del 26 de marzo de 2014, y que esta Carta Circular podía ser consultada en el vínculo y se abstuvo de dar otra opinión diferente: https://www.superfinanciera.gov. co/descargas?com $=$ institucional $\&$ name $=$ pubFile1007286\&downloadname $=$ cc29_14. doc.

Por otra parte, el Banco de la República informó mediante el envío de un correo electrónico, que la posición de ellos se encuentra expresada en el comunicado de prensa del 1 de abril de 2014, publicado en la página web, link http://www.banrep.gov. co/es/comunicado-01-04-2014, donde se refirió a las monedas virtuales (criptodivisa) en Colombia, y su posición no dista de lo manifestado por la Superintendencia Financiera.

Asobancaria, a través de un correo electrónico también expresó su punto de vista partiendo que las criptodivisas no ofrecen ventajas evidentes frente al dinero fiduciario y sí conllevan una serie de riesgos y desventajas.

\section{Conclusiones}

El uso de las criptodivisas en el mundo cada vez se está masificando, y el grado de incorporación en cada país va a depender del número de empresas que participen en este mercado, lo que hace que sea más dinámico. En el caso de Colombia, de los 7640 lugares registrados, tenemos un $0,3 \%$, porcentaje bastante pequeño, el cual es aún más pequeño teniendo en cuenta que solo nueve empresas tienen el servicio activo.

Aún en el mundo, se está legislando al respecto y varias naciones ven este sistema con buenos ojos, en Colombia los organismos gubernamentales encargados han adoptado una posición bastante conservadora, y hasta el momento no ven con buenos ojos este tipo de prácticas.

Por otra parte, podemos ver que en Colombia hay mucho por trabajar con relación a las criptodivisas, tanto en darlas a conocer, explicar su funcionamiento y su uso, así como dar a conocer sus ventajas y desventajas, para que de esta manera sean los diferentes negocios los que tomen la decisión de ponerlas o no en práctica, como una alternativa de pago, pudiendo minimizar los riesgos haciendo el cambio de las criptodivisa por una divisa real.

4 Asobancaria es la asociación representativa del sector financiero colombiano. Está integrada por los bancos comerciales nacionales y extranjeros, públicos y privados, las más significativas corporaciones financieras e instituciones oficiales especiales. 
Hablar de tan solo nueve empresas que tienen activo el servicio, del total de empresas que existen en Colombia, es casi pensar en un $0 \%$, y es claro, que por el mismo desconocimiento en el uso de este tipo de monedas, el flujo financiero es muy pequeño y de cierta manera "insignificante".

Colombia, no puede estar ajena ni alejada a la incorporación de esta nueva tendencia como alternativa al sistema monetario actual, debemos estar alertas a todo lo que se dé con relación a este tema en el mercado mundial, mirar qué se legisla al respecto, cómo sigue evolucionando en otros países, ya que no debemos nadar en contra de la corriente, y hay que dejar que los avances tecnológicos sigan su curso.

Para nadie es un secreto los riesgos que pueden existir de incertidumbre y volatilidad en el precio de las monedas virtuales, pero debemos dejar que las fuerzas de la oferta y la demanda jueguen su papel, y que nuevos actores formen parte de este nuevo mecanismo, con el fin de que se consolide y madure este mercado.

Predecir si bitcoin continuará reinando, o será reemplazado por alguna de las altcoin que estén o surjan en el futuro puede ser algo incierto, porque depende del gusto de los usuarios quien en últimas han sido los encargados de darle evolución y desarrollo a cada uno de estos tipos de monedas.

\section{Referencias}

Banco Central de Bolivia. (2014, mayo 6). Obtenido de Banco Central de Bolivia: https://www.bcb.gob.bo/webdocs/01 resoluciones/044\%202014.PDF

Bitcoin. (2016). Obtenido de Bitcoin: www.bitcoin.org

Bitcoin.org. (s.f.). Recuperado el 4 de marzo de 2016, de Bitcoin.org: https://bitcoin. org/es/faq\#por-que-los-bitcoin-tienen-valor

Bitcoinwiki. (2015, septiembre). https://en.bitcoin.it/wiki/. Obtenido de https:// en.bitcoin.it/wiki/Category:History

Bitcoinx. (2014, mayo 22). http://www.bitcoinx.com. Obtenido de http://www. bitcoinx.com/bitcoin-pizza-day-is-may-22-destined-to-become-the-first-bitcoinholiday/

Blockchain. (2016, junio). https://blockchain.info. Obtenido de https://blockchain. info/es/charts/market-price?timespan=all

Blockchainusa. (2014, diciembre 14). http://blockchainusa.org. Obtenido de http:// blockchainusa.org/\#/video-details

Chacón, P. (2014, febrero 27). El diario. Obtenido de www.eldiario.es: http://www. eldiario.es/hojaderouter/seguridad/ethereum-criptomoneda-hacker-desbancarBitcoin_0_276122447.html 
Chaum, D. (1985, octubre). Security without identification: transaction systems to make Big Brother obsolete. Communications of the ACM, 28(10), 1030-1044.

Coindesk. (2013, noviembre 12). http://www.coindesk.com. Obtenido de http://www. coindesk.com/bitcoin-accepting-subway-sandwich-shop-discovered-us/

CoinDesk. (2016). Obtenido de Coindesk: www.coindesk.com

Coinmap. (2016, marzo 18). https://coinmap.org. Obtenido de https://coinmap.org

Coinmarketcap. (2016, marzo 18). Obtenido de coinmarketcap.org: http:// coinmarketcap.com/all/views/all/

Criptoeconomia. (2015, febrero). http://criptoeconomia.blogspot.com.co. Obtenido de http://criptoeconomia.blogspot.com.co/2016_11_01_archive.html

Díaz, J. (2014, enero 10). Instituto Nacional de Ciberseguridad. Obtenido de www. incibe.es

Edwards, J. (2013, diciembre 4). http://www.businessinsider.com. Obtenido de http://www.businessinsider.com/220-million-sheep-marketplace-bitcoin-theftchase-2013-12

El Bitcoin. (2013, febrero 25). Obtenido de www.elbitcoin.org: http://elbitcoin.org/ ripple-competencia-o-complemento-de-bitcoin/

El Bitcoin. (2015, febrero 18). http://elbitcoin.org. Obtenido de http://elbitcoin.org/ bitcoin-es-la-peor-inversion-de-los-ultimos-anos/

El Diario. (2013, octubre). http://www.eldiario.es/. Obtenido de http://www.eldiario. es/turing/Silk-Road-supermercado-drogas-internet_0_183731705.html

Euribor. (2014, septiembre). http://www.euribor.com.es. Obtenido de http://www. euribor.com.es/foro/economia-bolsa-y-actualidad/17542-bitcoin-esto-a-traercola-20.html

Europapress. (2011, junio 16). http://www.europapress.es. Obtenido de http://www. europapress.es/portaltic/internet/noticia-wikileaks-asocia-bitcoin-mientras-leroban-medio-millon-dolares-20110616094507.html

Gómez, L. (2014, marzo 24). Semana.com. Obtenido de http://www.semana.com/ tecnologia/novedades/articulo/que-tan-legitimo-es-invertir-en-bitcoins/381503-3

Gómez, L. (2014, marzo 24). Revista Semana. Obtenido de www.semana.com

Higgins, S. (2014, julio 25). Coindesk. Obtenido de Coindesk: www.coindesk.com

History of Bitcoin. (2012). Recuperado el 24 de febrero de 2016 de http:// historyofbitcoin.org/ 
InfoCuria. (2015, octubre 22). Obtenido de Jurisprudencia del Tribunal de Justicia: http://curia.europa.eu/juris/document/document. jsf? text $=$ bitcoin\&docid $=$ $170305 \&$ page Index $=0 \&$ doclang $=$ es $\&$ mode $=$ req $\&$ dir $=\&$ occ $=$ first $\&$ part $=1 \& \operatorname{cid}=757798 \# \operatorname{ctx} 1$

Instituto Nacional de Ciberseguridad. (2014, noviembre 22). https://www.incibe.es. Obtenido de https://www.incibe.es/blogs/post/Security/SecurityBlog/Article and_comments/bitcoin_funcionamiento

Litecoin.org. (2011, octubre 7). Obtenido de www.litecoin.org: https://litecoin.org/es/

Mundodogecoin. (2014, noviembre 20). Obtenido de http://mundodogecoin.blogspot. com.co/2014/11/historia-de-dogecoin.html

Nakamoto, S. (2008). Bitcoin.org. Obtenido de https://bitcoin.org/bitcoin.pdf

Normas Brasil. (2013, octubre 10). Obtenido de http://www.normasbrasil.com.br/ norma/lei-12865-2013 260487.html

Oro y Finanzas. (2014, julio 14). Obtenido de www.oroyfinanzas.com

Patricia Peck Pinheiro Abogados. (2014, noviembre 21). Obtenido de www.felaban. net/archivos_memorias/archivo20141128145231PM.pptx

Raskin, M. (2013, noviembre 18). Obtenido de www.bloomberg.com

Siles, V. (2014, marzo 28). Obtenido de https://revistaitnow.com/bitcoin-unrecorrido-por-su-historia/

Superintendencia Financiera de Colombia. (2014, marzo 26). Obtenido de https:// www.superfinanciera.gov.co/jsp/loader. sf?1Servicio $=$ Publicaciones $\& 1$ Tipo $=$ publicaciones \& 1 Funcion $=$ load Contenido Publicacion $\&$ id $=10082781 \& \mathrm{~d}$ Print $=1$

Tecnología Bitcoin. (2014, mayo). Obtenido de http://tecnologiabitcoin.com/historiadel-bitcoin-2011/

Velasco, J. (2013, abril 12). Obtenido de hipertextual.com: http://hipertextual. com/2013/04/ripple-red-p2p-de-credito 